\title{
Inspection and control system for experiments in space robotics
}

DOI: $10.46932 / \operatorname{sfjdv2n3-023~}$

Received in: May 1st, 2021

Accepted in: Jun 30th, 2021

\author{
Glaydson Luiz B Lima \\ ITA, Depto. de Eng. Eletrônica e Computação, \\ DCTA, São José dos Campos, SP, Brazil \\ glaydson.bertozzi@gmail.com \\ Osamu Saotome \\ ITA, Depto. de Eng. Eletrônica e Computação, \\ DCTA, São José dos Campos, SP, Brazil \\ Ijar M. da Fonseca \\ ITA, Aerospace Engineering, DCTA, \\ São José dos Campos, SP, Brazil
}

\begin{abstract}
The communication subsystem is one among the various subsystems of a telerobotic space system. It is responsible for coordinating the commands received from the teleoperator control subsystem to the robotic arm, for reading signals from the sensors, and for stating the communication of the telerobot with the ground station. The telerobotic experiment under development by the ITA space robotics research group was developed with the purpose of investigating a robotic space system dynamics and control, including the study of the working and integration of all subsystems involved in the teleoperation control. The lab experiment consists of two identical units of robot manipulators, each of them mounted on its own floating air-supported platform. The objective is to simulate computationally the operations of rendezvous and capture in the microgravity' orbital environment, emulated by the floating manipulators' dynamics. The closed circuit for this system involves the in time position detection, transmission and data processing by using a position-tracking (X, Y, and Z) computer system combined with a Kinect sensor (RGB-D). The computer system comprises two computers capable of processing the positional images with greater accuracy. One of them receive and send the sensor data to a second computer which performs the data processing by proper algorithms in Matlab ${ }^{\circledR}$ and Simulink and sends commands to the robotic arm via WIFI (UDP protocol) network. The robot receives and executes the control signals moving the robotic arms whose position is again detected by the kinect sensor and informed back to the computer system, closing the control mesh and allowing the safe capture of the target. This work deals with the communication subsystem of the space robot experiment and its ability to set an integrated and efficient communication satisfying the telerobot control requirements
\end{abstract}

Key-words: Computer Vision, Robotics, Space, Microgravity, RGB-D, System of Communication, Aero Platforms, Network, Sensors.

\section{INTRODUCTION}

Space exploration has made intense use of robotics since the beginning of the conquest of space. All satellites were in fact robotic craft programmed to send data to Earth. In the early space age, spacecraft 
were programmed to send information about the upper atmosphere, the Earth's magnetic field and the topography of the Moon, Venus and Mars. The first probes were impact ones, developed to send images and videos of the Moon before crashing on the surface. The goal was to study the topography to plan the landing of man on the Moon. The impact probes were followed by the landing spacecraft. It was time to try the landing on the Moon since the the impact probes had allowed the necessary knowledge of Moon surface so as the landing area could be defined safely. The robotic probes' landing on the Moon was the last phase before them man landing on the Moon, that was accomplished successfully in 1969. Meanwhile probes was send to the Venus first as orbiters then as lander. Venus's atmosphere and temperature was studied increasing the knowledge of the planet. Later surface robots were built to operated on the dustcovered surface of the Moon and the rocky surface of Mars. Mars rovers sent chemical composition of the atmosphere and soil, analyze the existence of water in the subsoil, seek information about the possibility of life on the neighboring planet. These operations would not be possible without robots with the ability to operate automatically and autonomously (Sutantyo, D., 2015, Hara, S., 2006) in space and surfaces of the Moon, Venus and Mars. The conquest of the Moon and Mars go through orbital rendezvous and docking operations and berthing as well. Initially such operations were performed manually by astronauts, first during on-orbit tests and later in the mission that took man to the moon in 1969. Today, $\mathrm{RVD} / \mathrm{B}$ missions that provide services in orbit are unmanned but telerobotic. In spite of being unmanned the success of the robotic operations still rely on interaction of the on-board software with ground stations and/or data relay satellite. Such spacecraft work on the base of on-board software that implements risk phases of RVD/B automatically. The process automation and autonomy in the RVD/B involve new technologies of sensors and actuators and recently, artificial intelligence (AI). In this sense the more used methodology are Machine learning, deep learning, computer vision and so on, all supported by AI tools. The RVD/B are high-risk operations, because in the space environment, bodies experience an apparent weightlessness (because of the microgravity environment). A spacecraft-like robot manipulators and satellite mounted manipulators face two critical phases during the RVD/B: one occur during the close proximity immediately before the berthing or docking with the target. Berthing operations use robot manipulators' end effectors to capture the target while docking operations mate a docking port for the connection with the target. Both operations represents the second phase, when the chaser spacecraft implements the docking (mate with the docking port) or the berthing operation (using the end effectors to grasp a prescribed device) (Fehse, W. 2003). Any error in the mating with the docking port or with the grasping point (using robot arm's end effectors) may lead to loss of RVD/B mission. In the microgravity environment robot like-spacecraft behave like a free-flying objects. In the orbital configuration in which the spacecraft mounted manipulator move the end effectors (joint angles and center of mass displacement) 
toward the target, the whole system becomes sensitive to the efforts associated to the control signal commanded to the manipulator joints. This is to say that the whole system moves back in the contrary direction of the end effectors movement. The rotational motion (attitude motion) also is affected by the reaction torque associated to the torque commanded to joints. The critical risk is the loss of the target's docking port or the target grasping device. Two different action needs to be taken to avoid the loss risk: one is to compensate the displacement back of the whole system in the trajectory generation for robot arm end effectors and the other is to implement the synchronization and attitude stabilization of both systems (the chaser and the target) (Longman, R.W. 1994). For the RBD/B close approach the relative motion and the relative attitude must be considered in the system dynamics analysis (Gauthier, D., 1987, Ray, A. A. and Roppel, T. A., 2005), Jiang, L. 2012). In this regard, the experiments and laboratory on ground are fundamental for the simulation and validation of control algorithms via tests. As the microgravity environment can only be obtained in the laboratory for a very short time (between 4 to 8 seconds, in microgravity towers), one of the solutions found was experiments with floating robot platforms. Actually the platforms do not float, but operate almost frictionless on a thin (4 micron) air cushion. In this configuration, the platform become non-inertial robotic base because it moves, in reaction to the movements of the link joints of the manipulator, causing operational problems, especially when a target needs to be grabbed and moved from one position to another. This is typical of in-orbit assembly when a target structure is grasped and moved to a docking port in a station being mounted in orbit or when a satellite must be grasped to allow on-orbit servicing. At ITA, an experiment has been developed aiming at testing algorithms used for computer simulations of the control of RVD/B orbital operations (Da Fonseca, I. M. at all, 2015). The experiment involves two air-supported wireless robot manipulators that operates on a glass table almost frictionless by using compressed air. In the experiment, the communication system plays an extremely important role for the control tests of the relative rotationaltranslational motions of the air-supported manipulators during close approach and berthing operations. This communication system is the main goal of this paper. This type of experiment is sometimes referred as microgravity experiment because the floating manipulators have a dynamics behavior similar to that of orbital robot manipulators in operations of capture of on-orbit targets.

\section{ROBOT PRESENTATION AND DISCUSSION}

The position tracking computational system proposed in this article uses computational vision associated with Deep Learning (Das, B. 2016). The robot (Figure 1) is 6 degrees of freedom robotic arm. This robotic arm is mounted on to sliding air-supported platform and operates on a balanced glass table. The air is compressed and flows through the micro holes of three symmetric circular shoes disposed on 
the vertices of an equilateral triangle. The pressurized air counterbalances the gravity effect and the platform slide o air almost frictionless on a glass table. In this way, the floating platform moves in rotation (about its $\mathrm{z}$-axis) and translation (planar motion in $\mathrm{x}$ and $\mathrm{y}$ directions) while the platform mounted manipulator that translates and rotates with the platform executes 6 degrees-of-freedom through its six revolute joints, of which 5 joints perform rotations normal to the rotational motion of the platform and 1 degree-of-freedom of the end effectors to grasp a tool. The movement is analyzed under the premise that all dynamics take place on a non-fixed microgravity platform. Therefore, with this experiment it is possible to verify the impact of the movement of the mechanical arm on the floating mobile platform similar to that which occur in orbit with spacecraft-like robot manipulator considering the orbital planar $\mathrm{x}-\mathrm{y}$ motion (for the platform's translational motion). Furthermore, it is possible to test coupling strategies in the form of algorithms that result in the coupling of two robots with these same configurations. This scenario takes place in space, where two spacecraft, apparently weightless, approach and dock or berth (orbital operations of Rendezvous and docking/berthing)

Fig. 18 degrees-of-freedom Sliding robot manipulator system

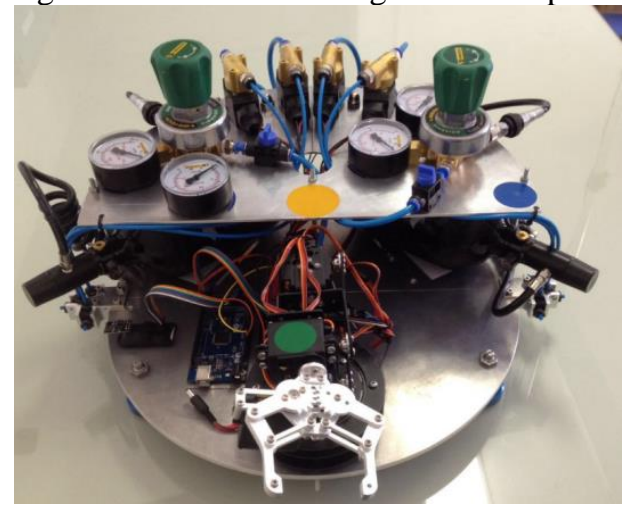

Each of the robotic system has two compressed air actuators, with three compressed air jet directions each. The actuator can execute and control the translational motion in $\mathrm{x}$ and $\mathrm{y}$ directions by using properly the jet directions. The combination of the jets in reverse an direct (x or y) directions allows the execution and control of the robotic platform rotational motion. Also it is possible to combine two sets of jets indirection, say -x and $y$ to generate the rotational motion of the platform.

Fig. 2 First and second figures illustrates one of the actuators and the respective air jets directions to execute and control the translational and rotational motions. The third figure illustrates one of the shoe with micro holes and air pressurized air that counterbalance the gravity, generating a microgravity conditions for the platform.
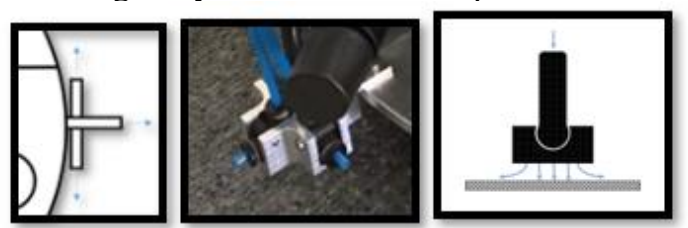
Fig. 3 The two cylinders of compressed air mounted on the robot platform

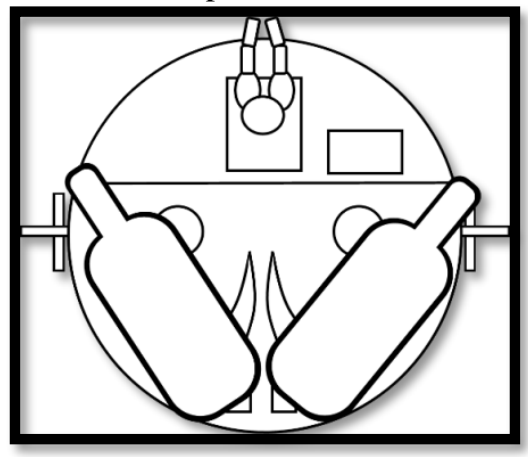

\section{INSPECTION AND CONTROL SYSTEM}

Position and direction movements are obtained by an image capture and processing system using the Kinect One sensor, manufactured by Microsoft. Figure 4 shows the positioning of Kinect in relation to the plane where the air-supported robot will operate.

Fig. 4 Figure 4: Kinect Sensor Positioning (RGB-D sensor)

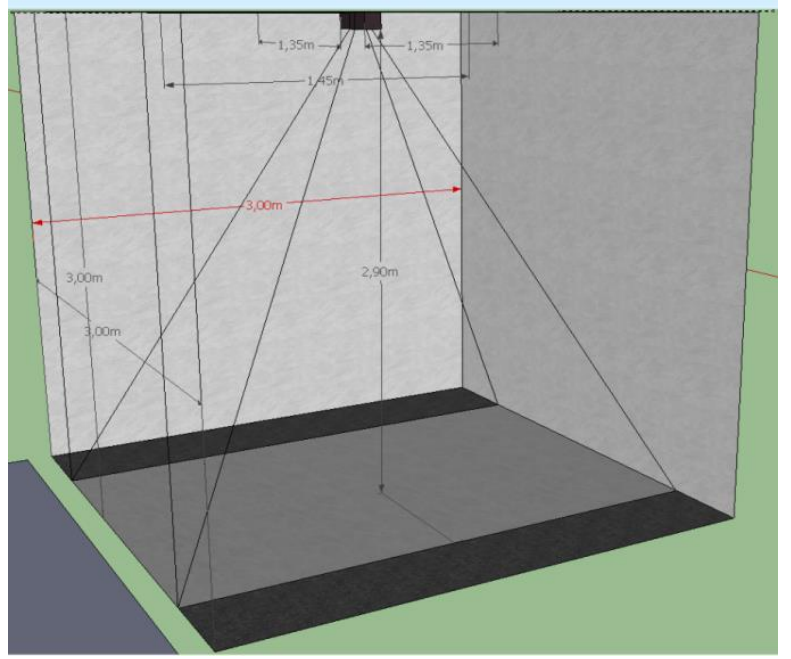

The Kinect sensor is composed of a set of cameras that capture the image (RGB) and depth (Depth) HD. The integration of data depth and color information results in a cloud of colored dots that contains approximately 300,000 dots in each frame. By recording RGB and depth images consecutively, a higher point density can be obtained, with acceptable accuracy (Lachat, E. at all, 2015, and Khoshelham, K. and 
Elberink, S. O., 2012), thus allowing to create a complete point cloud of an indoor environment, possibly in real time (Ruiz-Sarmiento, J.R., Galindo C., and Gonzalez-Jimenez J., 2014),. With this capture, the data is sent to a computer that analyzes the images and produces a record of the position, direction and speed of each of the robots in the experiment. Its location will be aided by round and colored labels that will aid the robot recognition process and its positioning. This information is sent to a second computer that controls the experiment. This computer has the logic that is being tested and, in possession of the captured information, adjusts the commands to be sent to the robots. Each robot receives commands through a WIFI connection via UDP protocol. These implemented commands and associated results are collected by the capture module (Figure 5).

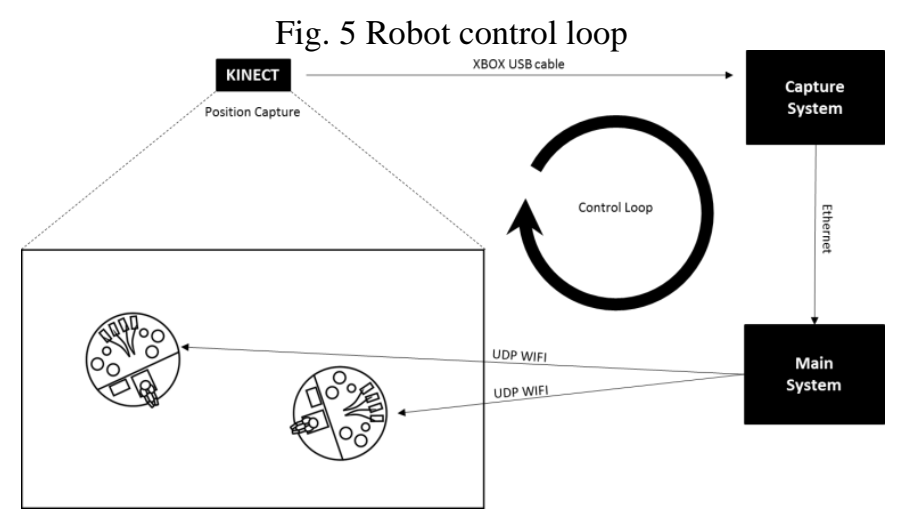

The capture system must have some functionalities so that it can operate properly in order to provide sufficiently precise information to effectively control the robots' movements. The basic features of the capture system are listed below

- Identify colored markings placed on the table, in robots and articulated arms (Figure 6). A identification of brands is carried out through computer vision techniques and processing of images. Hough's algorithm is applied to detect circular shapes, apply the algorithm of Sobel and then apply the detection algorithm of RGB colors. When detecting the regions of interest are calculated the centroids of each colored circle of interest (red, blue and yellow), drawn a point on the centroid and tracking of the $\mathrm{X}, \mathrm{Y}$, and $\mathrm{Z}$ coordinates with respect to these points, which allows you to have the proper positioning of the entire area, robot position and displacement of robotic manipulators. 
Fig. 6 Colored markings for identifying the position, direction and positioning of the robot arm

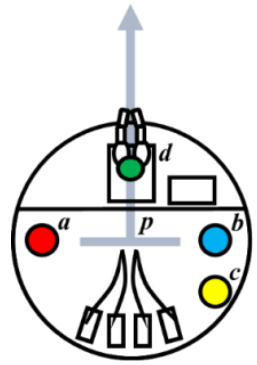

Identify colored markings placed on the table, robots and articulated arms (Figure 6). The identification of brands is carried out through computer vision techniques and processing of images. Hough's algorithm is applied to detect circular shapes, apply the algorithm of Sobel and then apply the detection algorithm of RGB colors. When detecting the regions of interest, the centroid of each colored circle of interest (red, blue and yellow), drawn a point on the centroid and tracking of the $\mathrm{X}$, Y, and Z coordinates with respect to these points is started. That allows to have the proper positioning of the entire area, robot position and displacement of robotic manipulators.

- Calculate the position and direction of the base of the robots in relation to the table. The robot position $\mathrm{p}$ is the midpoint between $\mathrm{a}$ and $\mathrm{b}$.

- Determine the robot direction, defined by the vector $\vec{u}=(c, b)$

- Identify the d position of the robotic arm of each robot. The robot's hand has a colored mark whose

- $\quad$ position is compared with the robot's position and its

- respective direction to identify its relative position

- to the base of the robot.

- Send the signal packets via UDP protocol. Sensors that capture light signals(lumens), light intensity (luxs) and ultraviolet rays (UV) of the environment. The variation of these signals changes the accuracy of coordinates. That's why these signs are captured and sent to the control system.

- Send data packets from positioning for the control system (connection Ethernet). This information is used to compose the package of data to be sent to the control system.

Fig. 7 generated by the capture system

\begin{tabular}{|c|c|c|c|c|c|c|c|c|c|}
\hline \multicolumn{10}{|c|}{120 bytes } \\
\hline 1 & 16 & 16 & 16 & 4 & 16 & 16 & 16 & 8 & 8 \\
\hline
\end{tabular}




\begin{tabular}{|c|l|}
\hline Part & \multicolumn{1}{|c|}{ Description } \\
\hline 1 & Sensor data \\
\hline 2 & Robot position 1 (double, double) \\
\hline 3 & Position and Direction of Robot 1 (double, double) \\
\hline 4 & Robot 1 arm position (double, double) \\
\hline 5 & Reading indication for robot 2 (int) \\
\hline 6 & Robot position 2 (double, double) \\
\hline 7 & Robot 2 direction (double, double) \\
\hline 8 & Robot arm position 2 (double, double) \\
\hline 9 & Time stamp \\
\hline 10 & Checksum \\
\hline
\end{tabular}

When the control system receives data from the sensor network, Deep Learning techniques are applied with the signals so that the system can maintain historical data and learn the appropriate correction levels to maintain the best image processing, allowing for better tracking precision and automatic adjustment to test environment conditions. In this way, the computer system receives all the data from the environment, applies filters and calibration, and when it receives the robot's actual positioning, it maintains greater coherence between the real environment and the one processed by the robot's navigation system. Allowing then to couple the two robots once the speed, position and distance can be determined values of the two robotic manipulators already considering the variations of local physical behavior (light, UV rays, etc). With this information, the control system implements algorithms in order to correctly perform the coupling of the two robots

The basic commands of the control system are listed below.

- Calculate the speed and direction of a robot's movement.

- Calculate the distance between robots.

- Calculate the alignment of the directions of the arms of the robots.

- Activate the compressed air actuators' air jets (Figure 8).

These commands are identified with the package of I data for robot identification, $\mathrm{L}$ or $\mathrm{R}$ respectively for the left and right sets, $f$ forwards, $r$ backwards and $s$ to the side. The desired activation time is also informed. The initial letter $\mathrm{T}$ identifies the command air jet drive.

Fig. 8 Data package for air jet drive

\begin{tabular}{|c|c|c|c|c|}
\hline \multicolumn{5}{|c|}{ 18 bytes } \\
\hline 2 & 4 & 2 & 2 & 8 \\
\hline$T$ & $1 \mid 2$ & $L \mid R$ & $F|R| S$ & time \\
\hline
\end{tabular}

- Move the robotic arms (Figure 9). The arm movement command is performed informing the angle of each of the 6 degrees-of-freedom of the arm beyond the desired time for the movement.

- Send actuation commands to robots via UDP protocol (WIFI connection). 
Fig. 9 Data package for robot robotic arm movement

\begin{tabular}{|c|c|c|c|c|c|c|c|c|}
\hline 2 & 4 & 8 & 8 & 8 & 8 & 8 & 8 & 8 \\
\hline$B$ & $1 \mid 2$ & $a 1$ & $a 2$ & $a 3$ & $a 4$ & $a 5$ & $a 6$ & time \\
\hline
\end{tabular}

With these actuation commands, it is possible to position and rotate the robots on the table (Figure 10) in order to obtain the coupling. The result of the movement is perceived by the capture system that feeds back to the control system.

Fig. 10 Positioning of robots on the table

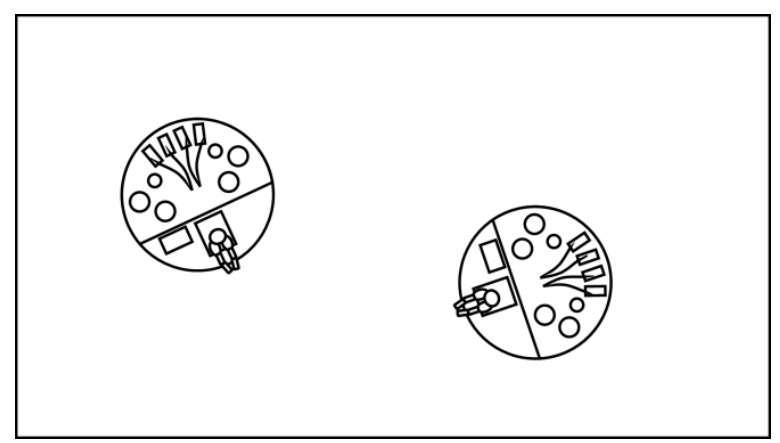

The control algorithm must implement some actions that are fundamental for carrying out the coupling strategies, shown below

- Implementation of the control loop with the reception of input information through listeners, blocks responsible for continuously receiving information about the capture system.

- Implementation of a decision tree identifying situations that deserve to be triggered

- events related to the coupling maneuver.

- Implementation of events and their associated routines that are part of the docking maneuver or mission success or failure situations.

- Implementation of a state model that determines at which stage the coupling process takes place. According to the strategy being tested, a new coding should be performed based on this macro view of the coupling solution. The robot has a very simple processing unit and is only used to perform the actions received by the control system. The robot's processor is a microcontroller (Arduino Mega) that is connected to a servo motor control module. The microcontroller (Arduino) is connected to the electronic actuation valves for activating the air jets of the actuators (figure 3). The commands received are identified and immediately executed by the arduino that activates the jets or sends the control command to the control module of the servo motors that control the robotic arm's 6 degrees-of-freedom. The control module receives the command with information on the desired angles for each of the arm servo motors as well as the desired time for the movement to be performed. All the logic and strategies regarding obtaining 
these robotic arm angles are related to the coupling algorithm that is not part of this work. The objective is to create the necessary environment for these strategies to be studied and elaborated in the future. In this way, the operational cycle of the experiment is completed once the capture, control and performance aligned with the objectives of the coupling mission are synchronized and integrated in a single system, creating a set of THE resources suitable for the study of robotic arm movements on a non-inertial (mobile) base that simulates the typical operations of RVD/B missions in orbit.

\section{CONCLUSION}

This article presents the inspection and control system of the translational-rotational movement of a robotic system composed of two air- supported manipulators supported by a thin layer of air (around 4 microns) provided by compressed air cylinders. The system of floating robots over air allows to computationally simulate operations typical of RVD/B space missions, especially in the final part of those operations, known as close proximity approximation and docking or berthing. In this scenario, the communication system presented in this article is fundamental to implement the control operations and guarantee the coupling between the two handlers. 


\section{REFERENCES}

Da Fonseca, I. M. at all (2015), Interaction Between Motions of Robotic Manipulator Arms and the NonFixed Base in On-Orbit Operations, J. Aerosp. Technol. Manag., São José dos Campos, Vol.7, No 4, Pages 443-453

Das, B. (2016). MRoCS: A new multi-robot communication system based on passive action recognition, Robotics and autonomous systems, Vol. 82, August 2016, Pages 46-60.

Fehse,W. (2003), Automated Rendezvous and Docking of Spacecraft, Cambridge University Press, New York, Pages 8-26.

Gauthier, D. (1987) Interprocess communication for distributed robotics. IEEE Journal of Robotics and Automation [0882-4967], vol. 3 fasc: 6, pp: 493-504.

Hara, S. (2006). New paradigms in wireless communication systems. Wireless Personal Communications [0929-6212], vol. 37, fasc: 3, Pages: 233-241.

Longman, R.W. (1994), Tutorial overview of the Dynamics and Control of Satellite-Mounted Robots, in Steven B. Skaar and Carl F. Ruoff, Teleoperation and Robotics in Space, Pages 237-258, Progress in Astronautics and Aeronautics, Vol.161

Jiang, L. (2012), Stable formation control of multi-robot system with communication delay. International journal of advanced robotic systems [1729-8806], vol. 9, Issue 1, Pages 1-10.

Khoshelham, K. and Elberink, S. O. (2012), Kinect Depth Data Accuracy and Resolution for Internal Mapping Applications, Journal Sensors.

Ray, A. A. and Roppel, T. A. (2005) Cooperative robotics using wireless communication. Thesis (M.S.) - Auburn University, 2005.

Lachat, E., Macher, H., Landes, T., and Grussenmeyer, P (2015) Evaluation and Calibration of an RGBD Camera (Kinect v2 Sensor) Towards a Potential Use for Short-range 3D Modeling, Journal Remote Sensing, Vol. 7, Pages 7, 13070-13097.

Ruiz-Sarmiento, J.R., Galindo C., and Gonzalez-Jimenez J. (2014), Experimental Study of Kinect Range Camera Performance for Mobile Robotics, Technical Report.

Sutantyo, D. (2015) Decentralized underwater multi-robot communication using bio-inspired approaches. Artificial Life and Robotics [1433-5298], vol: 20 fasc: 2 Pages: 152 -158. 